References:

Table. Case control studies

\begin{tabular}{lcccc}
\hline Region (Year) & Diagnosis & TB with MTX & TB without MTX & Odds Ratio \\
\hline Mexico (1999) & Mixed (4 RA) & $1 / 6$ & $5 / 75$ & 2.76 \\
Japan (2004) & RA & $3 / 47$ & $17 / 154$ & 0.56 \\
Canada (2009) & RA & $29 / 483$ & $21 / 1046$ & 3.12 \\
Brazil (2010) & Lupus & $2 / 3$ & $1 / 57$ & 112 \\
Taiwan (2012) & Psoriasis & $33 / 144$ & $464 / 2341$ & 1.2 \\
Taiwan (2015) & JIA & $4 / 357$ & $4 / 1026$ & 2.90 \\
South Africa (2017) & RA & $0 / 134$ & $0 / 18$ & NA \\
\hline
\end{tabular}

JIA=juvenile idiopathic arthritis

Acknowledgments: Funded by the International League Against Rheumatism and McGill University Global Health Scholar Awards

Disclosure of Interests: Anna Davidson: None declared, Alize Gunay: None declared, Ines Colmegna: None declared, Diane Lacaille: None declared, Hal Loewen: None declared, Michele Meltzer: None declared, Rosie Scuccimarri: None declared, Yewondwossen Mengistu: None declared, Sasha Bernatsky: None declared, Carol Hitchon Grant/research support from: UCB Canada; Pfizer Canada

DOI: 10.1136/annrheumdis-2020-eular.1544

\section{FRI0065 CLINICAL FEATURES OF METHOTREXATE ASSOCIATED LYMPHOPROLIFERATIVE DISORDER IN RHEUMATOID ARTHRITIS PATIENTS AND INFLUENCE OF CD8 POSITIVE LYMPHOCYTE} INFILTRATION

T. Kameda ${ }^{1}$, S. Nakashima ${ }^{1}$, M. Inoo ${ }^{2}$, I. Onishi ${ }^{2}$, N. Kurata ${ }^{2}$, H. Shimada ${ }^{1}$, M. Mahmoud Fahmy Mansour ${ }^{1}$, R. Wakiya ${ }^{1}$, M. Kato ${ }^{1}$, Y. Ushio ${ }^{1}$, K. Sugihara ${ }^{1}$, T. Miyagi ${ }^{1}, \mathrm{H}$. Dobashi ${ }^{1}{ }^{1}$ Kagawa University, Department of Internal Medicine, Division of Hematology, Rheumatology and Respiratory Medicine, Kagawa, Japan; ${ }^{2}$ Utazu Hospital, Kagawa, Japan

Background: Lymphoproliferative disorders (LPD) that develop in rheumatoid arthritis (RA) patients treated with MTX (MTX-LPD) is one of the important complications for RA patients. We have previously epidemiologically demonstrated an association between MTX and the development of LPD in RA patients ${ }^{1)}$. MTX-LPD has varied pathologies including various clinical symptom and histological finding. Therefore, we need more information about MTXLPD. In addition, it is one of the characteristics for MTX-LPD that spontaneous regression (SR) after MTX discontinuation. However, the mechanism of SR is not clarified.

Objectives: We collect the information such as clinical symptom and histological finding of MTX-LPD with RA patients, and clarify the clinical features of MTXLPD. In addition, we investigated the difference between SR cases and cases that treated with chemotherapy after MTX discontinuation (CTx cases).

Methods: We enrolled 90 MTX-LPD patients from Kagawa Prefecture, Japan between June 2005 and December 2019. Patients were diagnosed according to American College of Rheumatology (ACR) 1987 classification criteria or ACR/European League Against Rheumatism (EULAR) 2010 classification criteria, and treated with disease modifying antirheumatic drugs (DMARDs) including MTX. We collected as follow information; age, gender, duration of RA, laboratory data (lymphocyte counts and sIL-2R) and treatment of MTXLPD. In addition, we divided 16 MTX-LPD cases diagnosed histological into two groups (SR:CTx group; $n=10: 6$ ), and analyzed the histological findings (CD4, CD8, CD163 and CD47) using the staining in immunohistochemistry (IHC) between the two groups. Each positive cell analyzed using virtual viewer soft ImageScope.

Results: Characteristics of 90 MTX-LPD patients are as follow; mean age $66.5 \pm 11.2$ years, 63 female, duration of RA $18.5 \pm 19.4$ years. 65 patients $(72.2 \%)$ were spontaneously improved by discontinuing MTX. 58 patients $(64.4 \%)$ were proven MTX-LPD histologically. In these patients, diffuse large B-cell lymphoma (DLBCL) was the most frequent histological type of MTX-LPD (56.9\%). Infiltration of CD8 positive lymphocyte in the lesion was significant less in the SR cases than in the CTx cases (Figure 1). However, CD4, CD163 and CD47 positive cells had no significant difference between two groups.

Conclusion: We revealed clinical features of MTX-LPD with RA patients. In addition, CD8 positive lymphocytes are involved in tumor immunity. In this study, we suggested that the extent of CD8 positive lymphocyte infiltration may predict SR of MTX-LPD. Further study is necessary on revealing the mechanism of SR in MTX-LPD.

References:

[1] Kameda T. et al. Arthritis Care Res (Hoboken). 2014 Sep;66(9):1302-9.

Disclosure of Interests: None declared

DOI: 10.1136/annrheumdis-2020-eular.5309
FPO

Figure 1. CD8 positive lymphocytes in the specimen of lesion using the staining in immunohistochemistry (IHC) between SR and CTxgroup.

\section{FRI0066 SUBCLINICAL CORONARY CALCIFICATION ASSOCIATED WITH LONG-TERM CARDIOVASCULAR OUTCOMES IN RHEUMATOID ARTHRITIS}

G. Karpouzas ${ }^{1}$, S. Ormseth ${ }^{1}$, E. Hernandez ${ }^{1}$, M. Budoff ${ }^{1} .{ }^{1}$ Lundquist Institute of Biomedical Innovation, Torrance, United States of America

Background: Large, multicenter studies established the strong prognostic value of coronary artery calcium (CAC) scoring in asymptomatic individuals. Increasing CAC score is an independent predictor of worsening cardiovascular disease event risk in general patients. The prognostic significance of higher CAC score strata in the long-term cardiovascular risk in rheumatoid arthritis (RA) is unknown. Objectives: To evaluate the long-term cardiovascular event risk across CAC strata in a prospective, single center cohort of established RA patients without symptoms or prior diagnosis of cardiovascular disease.

Methods: One hundred-fifty patients underwent computed tomography angiography for coronary atherosclerosis evaluation. CAC score was measured according to Agatston. CVD events were prospectively recorded, including cardiac death, myocardial infarction, unstable angina, revascularization, stroke, claudication, and heart failure hospitalization over $6.0 \pm 2.4$ years of follow-up. Unadjusted robust Cox proportional hazards regression models evaluated CVD event risk across higher CAC strata $(C A C=1-99, C A C=100-399$ and $C A C \geq 400)$ compared to $\mathrm{CAC}=0$. Additional multivariable robust $\mathrm{Cox}$ regression models with time-varying covariates evaluated the impact of log transformed CAC or different CAC thresholds $(C A C>0$ vs. $C A C=0, C A C \geq 100$ vs. $C A C<100$ and $C A C \geq 400$ vs. $C A C<400)$

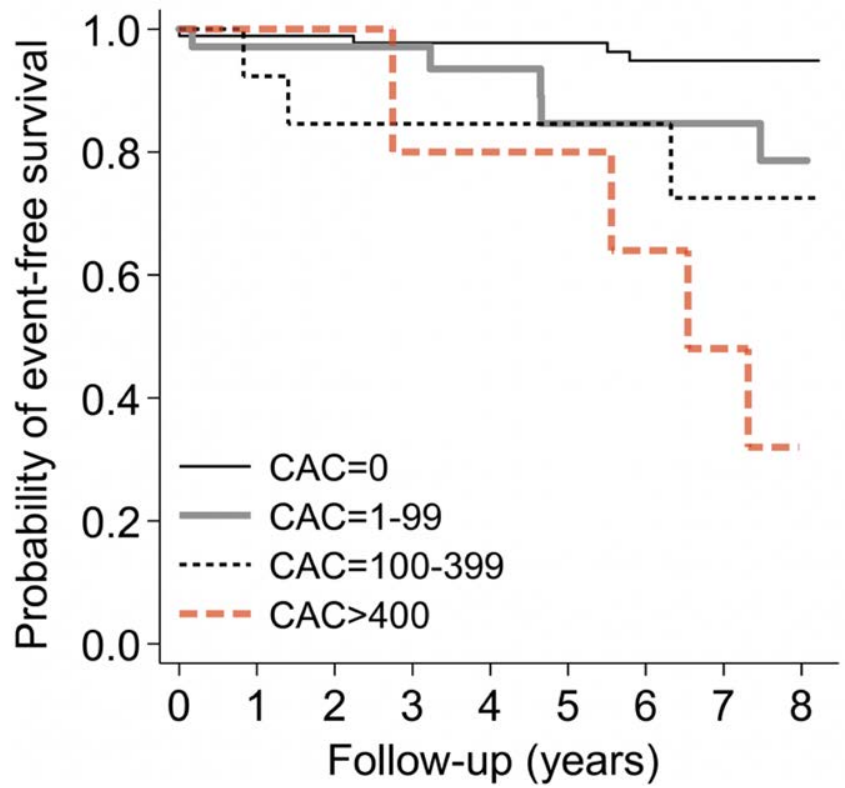

Figure 1. Increasing CAC scores associated with higher cardiovascular event risk in RA 
on future CVD events. Models were controlled for Framingham-D'Agostino clinical risk score, time-varying current bDMARD use and time-varying CRP.

Results: Sixteen patients incurred 19 events, for a total of 2.1 (95\% Cl 1.33.3) events/100 patient-years. Increasing HR for cardiovascular events was observed for ascending CAC strata; 3.87 (1.03-14.48), 6.31 (1.38-28.91) and 16.98 (4.50-64.10) for $\mathrm{CAC}=1-99, \mathrm{CAC}=100-399$ and $\mathrm{CAC} \geq 400$ respectively compared to $\mathrm{CAC}=0$ (figure 1 ). In fully adjusted models, $\mathrm{CAC}$ score associated with future event risk independently of Framingham D'Agostino score, time-varying bDMARD use and time-varying CRP ( $\mathrm{HR}=1.31$ [95\%Cl 1.04-1.66]). CAC thresholds $\geq 100$ (vs. $<100$ ) and $C A C \geq 400$ (vs. $<400$ ) in fully adjusted models similarly constituted independent predictors of long-term cardiovascular events (Figure 2).

\begin{tabular}{|c|c|c|c|c|}
\hline$C A C>0$ vs. $C A C=0$ & & \multicolumn{2}{|c|}{$\begin{array}{l}\text { Adjusted HR for CVD event } \\
\text { (95\% Confidence Interval) }\end{array}$} & p-value \\
\hline CAC & & $+\bullet$ & $2.74(0.69-10.96)$ & 0.153 \\
\hline Framingham-D'Agostino & & - & $1.09(1.05-1.14)$ & $<0.001$ \\
\hline Time-varying bDMARDs & $\longrightarrow$ & & $0.27(0.10-0.72)$ & 0.009 \\
\hline Time-varying CRP & & 6 & $1.09(0.81-1.46)$ & 0.582 \\
\hline \multicolumn{5}{|l|}{$C A C \geq 100$ vs. $C A C<100$} \\
\hline CAC & & $\longrightarrow$ & $3.17(1.09-9.20)$ & 0.034 \\
\hline Framingham-D'Agostino & & - & $1.10(1.06-1.14)$ & $<0.001$ \\
\hline Time-varying bDMARDs & $\longrightarrow$ & & $0.24(0.09-0.63)$ & 0.004 \\
\hline Time-varying CRP & & 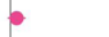 & $1.20(0.94-1.53)$ & 0.141 \\
\hline \multicolumn{5}{|l|}{$C A C \geq 400$ vs. $C A C<400$} \\
\hline CAC & & $\longrightarrow$ & $7.22(1.59-32.73)$ & 0.010 \\
\hline Framingham-D'Agostino & & - & $1.09(1.06-1.13)$ & $<0.001$ \\
\hline Time-varying bDMARDs & $\bullet$ & & $0.14(0.04-0.56)$ & 0.005 \\
\hline Time-varying CRP & & e & $1.16(0.91-1.47)$ & 0.235 \\
\hline 0.01 & 0.1 & 10 & 100 & \\
\hline
\end{tabular}

Figure 2. Impact of different CAC thresholds on cardiovascular event risk in RA

Conclusion: Increasing CAC scores are strong, independent predictors of longterm cardiovascular events in RA patients without symptoms or prior diagnosis of cardiovascular disease.

Disclosure of Interests: George Karpouzas Grant/research support from: Pfizer, Consultant of: Sanofi-Genzyme-Regeneron, Janssen, Speakers bureau: Sanofi-Genzyme-Regeneron, BMS, Sarah Ormseth: None declared, Elizabeth Hernandez: None declared, Matthew Budoff: None declared DOI: 10.1136/annrheumdis-2020-eular.5021

\section{FRI0067 ALL-CAUSE AND CAUSE-SPECIFIC MORTALITY OF PATIENTS WITH RHEUMATOID ARTHRITIS IN KOREA: A NATION-WIDE POPULATION-BASED STUDY}

E. Lee ${ }^{1}$, A. Shin ${ }^{2}$, J. Lee ${ }^{3}$, Y. J. Ha ${ }^{2}$, Y. J. Lee ${ }^{2}$, E. B. Lee', Y. W. Song ${ }^{1}$, E. H. Kang ${ }^{2}$. ${ }^{1}$ Seoul National University Hospital, Division of Rheumatology, Department of Internal Medicine, Seoul, Korea, Rep. of (South Korea); ${ }^{2}$ Seoul National University Bundang Hospital, Division of Rheumatology, Department of Internal Medicine, Seongnam, Korea, Rep. of (South Korea); ${ }^{3}$ Inha University Hospital, Prevention and Management Center, Incheon, Korea, Rep. of (South Korea)

Background: Rheumatoid arthritis (RA) is a chronic inflammatory disease associated with excess mortality.

Objectives: To compare all-cause and cause-specific mortality between RA patients versus general population, using a nationally representative cohort from Korea National Health Insurance Service database (KNHIS).

Methods: Patients with RA aged $\geq 40$ years were identified from 2010-2016 KNHIS database and their annual cohorts were constructed per each calendar year. RA patients of each cohort were required to have prevalent RA on January $1^{\text {st }}$ of the given calendar year. The KNHIS were linked with Korea national mortality data to obtain cause of death information and to estimate expected deaths of RA patients in reference to mortality data of the general population in Korea. Standardized mortality ratio (SMR) with a 95\% confidence interval $(\mathrm{Cl})$ was calculated to compare mortality of RA patients and general population.

Results: A total of 6,404 deaths occurred among 79,440 RA patients during 2011-2016 period, showing all-cause SMR (95\% CI) of 1.45 (1.42-1.49) compared to general population. The SMR was $1.72(1.59-1.85)$ for biologics users and $1.43(1.39-1.46)$ for non-users, versus general population. The annual SMR of RA patients showed a growing trend of mortality over time particularly among biologics users (Table 1). The SMRs for common causes of death among RA patients showed approximately tripled risk of dying due to pulmonary and infectious causes compared to general population (Table 2). There was approximately $20 \%$ increased risk of cardiovascular deaths but $8 \%$ decreased risk of cancer-related deaths among RA patients compared to general population (Table 2)

Table 1. Annual all-cause SMR of RA patients compared to 2010 general population

\begin{tabular}{lccc}
\hline Calendar year $^{1}$ & RA patients & Biologics users & Biologics non-users \\
\hline 2011 & $1.00(0.92-1.07)$ & $1.13(0.87-1.39)$ & $0.98(0.90-1.06)$ \\
2012 & $1.13(1.06-1.21)$ & $0.98(0.75-1.22)$ & $1.15(1.07-1.23)$ \\
2013 & $1.22(1.14-1.30)$ & $1.29(1.04-1.54)$ & $1.21(1.13-1.29)$ \\
2014 & $1.30(1.23-1.38)$ & $1.53(1.26-1.79)$ & $1.28(1.20-1.36)$ \\
2015 & $1.45(1.38-1.53)$ & $1.81(1.53-2.09)$ & $1.41(1.33-1.50)$ \\
2016 & $1.45(1.37-1.52)$ & $1.94(1.66-2.22)$ & $1.39(1.31-1.47)$ \\
\hline
\end{tabular}

$\mathrm{Cl}$, confidence interval; $\mathrm{RA}$, rheumatoid arthritis; SMR, standardized mortality ratio

${ }^{1} 2010$ data were used to identify RA diagnosis dates but were not included for SMR estimation since diagnosis dates of RA were unclear for those who had RA in 2010.

Table 2. Cause-specific SMR comparing RA patients versus general population

\begin{tabular}{lccc}
\hline Cause of death & RA patients & Biologics users & Biologics non-users \\
\hline Infection & $3.12(2.93-3.13)$ & $4.12(3.36-4.88)$ & $3.03(2.83-3.23)$ \\
Cancer $_{\text {Respiratory }}{ }^{1}$ & $0.92(0.87-0.97)$ & $0.98(0.81-1.14)$ & $0.91(0.85-0.96)$ \\
Cardiovascular $^{2}$ & $3.14(2.97-3.32)$ & $4.64(3.89-5.38)$ & $3.01(2.83-3.19)$ \\
\hline
\end{tabular}

$\mathrm{Cl}$, confidence interval; $\mathrm{RA}$, rheumatoid arthritis; SMR, standardized mortality ratio

${ }^{1}$ Includes pulmonary infections and chronic lung diseases (J00-J98, U04); ${ }^{2}$ Includes atherosclerotic and non-atherosclerotic deaths (I00-199).

Conclusion: The all-cause mortality of RA patients during 2011-2016 was overall $45 \%$ greater than that of general Korean population, which increased annually over the study period. The increased mortality was more prominent among biologics users than non-users. The risk of infectious and respiratory deaths were tripled among RA patients compared to general population.

References:

[1] Sokka T, et al. Mortality in rheumatoid arthritis: 2008 update. Clin Exp Rheumatol. 2008;26(5 Suppl 51):S35-61.

Acknowledgments: None

Disclosure of Interests: None declared

DOI: 10.1136/annrheumdis-2020-eular.5303

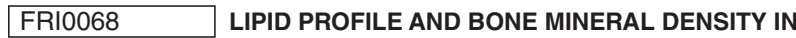 PATIENTS WITH RHEUMATOID ARTHRITIS: IS THERE AN ADDED RISK OF OSTEOPOROSIS?}

B. Touil ${ }^{1}$, H. Azzouzi ${ }^{1}$, O. Lamkhanat ${ }^{1}$, F. Chennouf ${ }^{1}$, I. Linda ${ }^{1} .{ }^{1}$ Centre Hospitalier Universitaire Mohammed VI, Rheumatology, Oujda, Morocco

Background: Bone is a target in many inflammatory rheumatic diseases such as rheumatoid arthritis (RA). It has been supposed that an atherogenic lipid profile could be associated with lower bone mineral density (BMD) and vertebral fractures (VF).

Objectives: We aimed to evaluate the relationship between the lipid profile, BMD and the presence of VF in RA patients.

Methods: A cross sectional study was conducted in a population of 169 established RA. In each subject we evaluated the body mass index (BMI), tobacco use, alcohol consumption, presence of diabetes and high blood pression, lipid profile (total cholesterol (TC), High density lipoprotein cholesterol (HDLc), low density lipoprotein cholesterol (LDLc), triglycerides (TG), and VF. RA characteristics were also assessed (disease duration, disease activity score (DAS), auto antibodies, corticosteroid intake, and secondary sjogren's syndrome). BMD was measured by dual energy X-ray absorptiometry (DXA) in lumbar spine and femoral neck. Logistic and linear regression were performed with SPSS 20, both BMD and VF were assessed as dependent variables.

Results: The mean age was $55.5 \pm 11.9$ years, with a female predominance (152 women). The average BMI was $26.79 \pm 5.36$. We had $24.3 \%$ of hypertensive patients and $16.6 \%$ of diabetics. The average lipid concentrations were $4.39 \pm 1$ $\mathrm{mmol} / \mathrm{L}$ for TC, $1.293 \pm 0.36 \mathrm{mmol} / \mathrm{L}$ for HDLc, $2.74 \pm 0.80 \mathrm{mmol} / \mathrm{L}$ for LDLc and $1.25 \pm 0.62 \mathrm{mmol} / \mathrm{L}$ for $\mathrm{TG}$. At the linear regression there was no correlation between plasma lipid concentrations and BMD, whether at the lumbar spine or the femoral neck. However we found a significant correlation between VF and high TC concentrations ( $p=0.043$, OR: $2.864,95 \%$ IC [1.036-7.922]). At the multivariate regression, high TC levels were still associated with VF, adjusted in BMI, age and the duration of corticosteroid use $(p=0.006$, OR: $6.07,95 \%$ 